\section{Biohazards of Prolonged N95 Use: Effects on Arterial Blood Gases, Peak Expiratory Flow Rate and General Well-being of Healthcare Professionals during COVID-19 Pandemic}

Sir,

Globally as millions of people have been staying at home to prevent human to human transmission, ${ }^{1}$ healthcare professionals (HCPs) stand at the front most, despite the unpredictable outcomes and daily evolving guidelines on coronavirus disease 2019 (COVID19). In these times, working as a frontline professional, where most part of day is spent in personal protective equipment (PPE) with $\mathrm{N}-95$ being constant part of face (as respirators are fundamental for HCPs in aerosol borne diseases handling), ${ }^{2}$ unusual symptoms were experienced and reported randomly, which included headaches, mood disturbance, fatigue and lethargy. These symptoms could be attributed to work-related stress but endemics and epidemics have not been a new experience for HCPs of the subcontinent. As the healthcare system has a very non-efficient mechanism of notifiable infectious diseases registration, starting from foundation year, most HCPs have faced the potential risk of exposure to various airborne/blood borne/waterborne endemics and epidemics, and are still exposed at various levels. Few notable exposures have been tuberculosis, and congo-crimean virus, rota virus, hepatitis viruses, vibrio cholerae, chickengunya, chickenpox, extremely drug resistant enteric fever and human immunodeficiency virus.

HCPs in Pakistan have developed a baseline subconscious stress coping mechanism of variable degree while working in such unfortunate situations, where odds of acquiring infection from workplace are always high. Fighting this global pandemic is not only an unprecedented challenge but has opened a Pandora box, where everything once considered safe is now under question mark. With our unusual experience of symptoms, we inquired about the actual biosafety of PPE by relooking into manuals provided by various prototype manufacturers. To our utter surprise, we failed to find any substantial data highlighting retention of noxious gases and effects on respiratory functions with use of PPE respirators/masks.

We recruited 75 HCPs (out of 110 approached) working in different COVID-19 setups all over Pakistan, wearing N95 respirator ensemble for the duration of more than 6 hours in our study. This included doctors, nurses and paramedics working as frontliners in COVID-19. Subjects with any physical or psychiatric diagnosis at the time of study were not included in the analysis. They were evaluated before and after their professional duty to study the effects of possible $\mathrm{CO}_{2}$ retention on development of related symptoms, peak expiratory flow rate values, variation in oxygen saturation observed on pulse oximeter, and long term changes on spirometry. $\mathrm{CO}_{2}$ retention usually manifests with the symptoms of headache, dizziness, lethargy, flushing, tachypnea, tachycardia and dyspnea ${ }^{3}$ and these variables were assessed in subjects after documenting basic demographic and clinical characteristics of the participants (age, gender, profession, allergies and comorbid conditions).

Among the study participants, $70(93.3 \%)$ experienced headaches, 50 (66.7\%) lethargy, 38 (50.6\%) dizziness, 23 (30.6\%) nausea, 18 (24\%) dyspnea and 12 (16\%) tachypnea. A variability of an average $25 \mathrm{ml}$ was observed with PEFR (normal average between $350-500 \mathrm{ml} / \mathrm{L})^{4}$ pre-and post-N95 use, keeping in mind the limitation that post duty fatigue impeded full capacity exhalation. While on duty oxygen saturation variation was documented in a range of $89-95 \%$ (measured using pulse oximetry). Last parameter that was employed in this study was to ascertain arterial blood gases (not all subjects consented to it). Fifty out of 75 consented for it. Inference deducted from the partially collected data on difference of pre- and post-duty $\mathrm{PCO}_{2}$ was between 0.3 to $0.7 \mathrm{kPa}$. Spirometry could not be performed in any subject due to potential hazards of exposure to aerosols and resource constraints.

The above mentioned results, that include the parameters of $\mathrm{CO}_{2}$ narcosis, variation in PEFR, difference in oxygen saturation, and general wellbeing of HCP's, reflect that use of this product needs scrutinising from biosafety point of view. It should be emphasised that there are no shortcuts; and quality control must never be compromised in urgency of launching the product. This letter has been written with a hope that it may help the authorities in regulating quality control of product, making it HCPs-friendly. A detailed policy is needed to implement safe duty hours duration with work breaks in between. Proper trials are needed to assess effects on blood concentration of $\mathrm{O}_{2}$ and $\mathrm{CO}_{2}$, pulmonary function tests and negative impacts on frontline HCPs health using N95, and similar masks in current pandemic and related situations. Appropriate authorities, like WHO, should direct all authentic manufactures in PPE to ensure that all essential biosafety parameters are addressed by proper trails and research prior to launch of the product. ${ }^{5}$ Thus, in the global response, the safety of healthcare workers must be ensured as a priority; and proper steps to betaken in this regard.

\section{CONFLICT OF INTEREST:}

The authors declared no conflict of interest.

\section{AUTHORS' CONTRIBUTION:}

MHA: Conceived the main idea and framework of this study design.

MHA, UBZ, UZ: Contributed in the collection and management of data; discussed result and contributed to writing, drafting and proofreading of final manuscript; collectively provided critical feedback and helped shape the analysis and manuscript. 


\section{REFERENCES}

1. Xu X, Chen P, Wang J, Feng J, Zhou H, Li X, et al. Evolution of the novel coronavirus from the ongoing Wuhan outbreak and modeling of its spike protein for risk of human transmission. Sci China Life Sci 2020; 63(3):457-60. doi:10.1007/s11427-020-1637-5

2. Artika IM, Ma'roef CN. Laboratory biosafety for handling emerging viruses. Asian Pac J Trop Biomed 2017; 7(5):483-91. doi:10.1016/j.apjtb.2017.01.020

3. Institute of Medicine (US) Committee on Personal Protective Equipment for Healthcare Personnel to Prevent Transmission of Pandemic Influenza and Other Viral Respiratory Infections: Current Research Issues, Larson EL, Liverman CT, eds. Preventing Transmission of Pandemic Influenza and Other Viral Respiratory Diseases: Personal Protective Equipment for Healthcare Personnel. Washington (DC): National Academies Press (US); 2011.

4. Dikshit MB, Raje S, Agarawal M J. Lung functions with spirometry: An Indian perspective-l. Peak expiratory flow rates. Indian J Physiol Pharmacol 2005; 49(1):8-18.
5. Lazzarino Al, Steptoe A, Hamer M, Michie S. Covid-19: Important potential side effects of wearing face masks that we should bear in mind. BMJ 2020; 369:m2003. doi:10.1136/bmj.m2003

Muhammad Hammad Athar', Usama Bin Zubair ${ }^{2}$ and Ujala Zakir $^{1}$

${ }^{1}$ Department of Medicine, Combined Military Hospital (CMH), Malir Cantt, Karachi, Pakistan

${ }^{2}$ Department of Psychiatry, Master Misericordiae University, Dublin, Ireland

Correspondence to: Dr. Usama Bin Zubair, Department of Psychiatry, Master Misericordiae University, Dublin, Ireland E-mail: drusamabinzubair@yahoo.com

Received: April 22, 2020; Revised: July 23, 2020;

Accepted: August 12, 2020

DOI: https://doi.org/10.29271/jcpsp.2020.JCPSPCR.CR134 